\title{
ANÁLISE DOS PARÂMETROS DO MODELO DE ADSORÇÃO EM MULTICAMADA, CALOR ISOSTÉRICO E ENTROPIA DE DESSORÇÃO PARA ISOTERMAS DE CEVADA
}

\author{
G. D. MAIA ${ }^{1 *}$, G. ALBINI ${ }^{1}$, J. T. FREIRE $^{1}$ \\ ${ }^{1}$ Universidade Federal de São Carlos, Departamento de Engenharia Química \\ e-mail:maia@ufscar.br
}

\begin{abstract}
RESUMO
A determinação de isotermas de adsorção e dessorção permite a obtenção de informações acerca da umidade de equilíbrio em materiais de interesse, além de permitir a análise da qualidade da água presente nesses sistemas e da energia necessária para a remoção dessa umidade, essencial para processos que envolvem secagem. Assim, o presente estudo visou a determinação experimental das isotermas de cevada em várias temperaturas com posterior análise de suas informações termodinâmicas fornecidas pelos parâmetros do modelo de adsorção em multicamada GAB, juntamente com os cálculos do calor isostérico líquido e da entropia de dessorção. Para a determinação das isotermas utilizou-se o método gravimétrico estático empregando soluções saturadas de atividade de água conhecida. As isotermas foram determinadas a $15^{\circ} \mathrm{C}, 20^{\circ} \mathrm{C}, 30^{\circ} \mathrm{C}$ e $50^{\circ} \mathrm{C}$ e apresentaram boa concordância com os dados fornecidos pela literatura até uma atividade de água de 0,4. O modelo GAB ajustou com precisão os dados experimentais obtidos para as isotermas de dessorção e seus parâmetros foram capazes de informar a presença de três classes de água adsorvidas no material. Os cálculos do calor isostérico líquido e da entropia de dessorção corroboraram as informações obtidas dos parâmetros do modelo GAB e permitiram a avaliação da umidade onde ocorre a formação da monocamada completa.
\end{abstract}

\section{INTRODUÇÃO}

A água é o principal componente associado ao processamento de cereais, frutas, folhas e gêneros alimentícios de uma maneira geral. Ela apresenta uma considerável influência nas variáveis de processamento, na caracterização e na estabilidade desses produtos (QUIRIJNS et al., 2005b).

Além de se conhecer sua quantidade, é necessário, também, conhecer o estado em que a água se encontra no material. Seu estado é estabelecido por uma complexa interação existente entre a água e os componentes do material, como proteínas, lipídios e carboidratos (QUIRIJNS et al., 2005b).
A relação fundamental que descreve a interação entre a água e o material onde ela se encontra é aquela que relaciona a atividade de água e o conteúdo de água presente nesse dado material, numa determinada temperatura e pressão, conhecida como isoterma de adsorção/dessorção de água.

Dados referentes à adsorção e dessorção formam a base para o cálculo das forças motrizes em operações de transferência de massa, como por exemplo, processos de secagem (KIRANOUDIS, et al., 1993).

Essas informações são de grande importância no processamento de cevada para a produção de malte cervejeiro (KUNZE, 2004). Nele, a cevada passa por etapas como o armazenamento, reumidificação e secagem, 
todas elas envolvendo o controle do teor de umidade dos grãos.

Nesse sentido, Vermuganti (1980) determinou os valores de umidade de equilíbrio de sementes de cevada num intervalo de temperaturas de $10^{\circ} \mathrm{C}$ a $40^{\circ} \mathrm{C}$ utilizando o modelo de Chung e Pfost para ajustar seus dados experimentais.

Ainda que os trabalhos presentes na literatura descrevam o comportamento de adsorção e dessorção para a cevada, nenhum dos estudos avançou no sentido de obter informações termodinâmicas relevantes.

Dessa forma, o presente trabalho visou a determinação experimental das isotermas de adsorção e dessorção para sementes de cevada inteiras a $15^{\circ} \mathrm{C}, 20^{\circ} \mathrm{C}, 30^{\circ} \mathrm{C}$ e $50^{\circ} \mathrm{C}$ e sua posterior análise utilizando o modelo de Guggenheim, Anderson e de Boer (GAB) (ANDERSON, 1946) analisando o significado físico de seus parâmetros.

Além disso, o presente trabalho teve como objetivo os cálculos de calor isostérico líquido e entropia de dessorção e sua comparação com as análises obtidas pela avaliação dos parâmetros do modelo GAB.

\section{TEORIA}

\section{1 Água livre e água ligada}

A determinação da classe da água presente em um material pode ser obtida através da análise das isotermas de adsorção e dessorção, que relacionam a atividade da água contida no material com a umidade de equilíbrio em uma dada temperatura e pressão.

A água ligada corresponde à porção da umidade que se encontra fortemente ligada às moléculas do material, enquanto a água livre corresponde àquela porção que apresenta pouca interação energética com as moléculas de material (KIRANOUDIS et al. 1993).

A atividade de água é definida pela razão entre a pressão parcial de vapor em equilíbrio da água presente no material e a pressão de vapor da água pura na mesma temperatura. Dessa forma, as isotermas de adsorção e dessorção permitem a avaliação do quão forte é a ligação entre a água e o adsorvato. (QUIRIJNS, et al. 2005a).

\subsection{Fundamentos teóricos do modelo GAB}

$\mathrm{O}$ modelo GAB representa uma extensão refinada das teorias de Langmuir e da extensão proposta por Brunauer, Emmett e Teller no modelo BET.

A base teórica para o modelo GAB é a consideração de adsorção física localizada em multicamada, sendo que a primeira camada de água cobre uniformemente a superfície do adsorvato e é fortemente ligada a ele, formando o que se chama de monocamada.

As moléculas dispostas na multicamada apresentam interações com o adsorvato em níveis que variam desde a monocamada até a água líquida, ou água de bulk (QUIRIJNS et al., 2005b).

Dessa forma, sucessivas camadas de água apresentam cada vez mais propriedades semelhantes às da água líquida (RAO, RIZVI e DATTA, 2005).

Três considerações são utilizadas para o desenvolvimento do modelo GAB: que o adsorvato apresente sítios independentes e distinguíveis de mesma atividade, isotérmicos e abertos ao vapor adsorvente. (ANDERSON, 1946).

A termodinâmica estatística conduz à expressão do modelo GAB, representada pela Equação 1.

$$
\frac{X}{X_{m}}=\frac{C_{g} \cdot K \cdot a_{w}}{\left(1-K \cdot a_{w}\right) \cdot\left(1-K \cdot a_{w}+C_{g} \cdot K \cdot a_{w}\right)}
$$

Nela, os três parâmetros do modelo $\mathrm{GAB}, \mathrm{C}_{\mathrm{g}}$, K e $X_{m}$ possuem significado físico.

$\mathrm{C}_{\mathrm{g}}$ é definido como a razão entre a função de partição da primeira molécula adsorvida em um sítio e a função de partição das moléculas adsorvidas além da primeira molécula na multicamada. É uma medida da 
força de ligação da água aos sítios de ligação primários e tem natureza intrinsecamente entálpica.

Assim, quanto maior o valor de $\mathrm{C}_{\mathrm{g}}$, mais fortemente a água estará ligada na monocamada e, consequentemente, maior será a diferença de entalpia entre as moléculas da monocamada e as da multicamada (QUIRIJNS et al., 2005b).

Considerando que as moléculas da monocamada apresentam pouca mobilidade e, consequentemente, um restrito número de configurações possíveis, o efeito entrópico associado a $\mathrm{C}_{\mathrm{g}}$ é menor do que o entálpico (VAN DER BERG, 1981).

$\mathrm{K}$ é definido como a razão entre a função de partição das moléculas de água líquida e a função de partição das moléculas adsorvidas na multicamada.

$\mathrm{O}$ conteúdo entrópico associado a $\mathrm{K}$ pode ser explicado considerando que as moléculas de água líquida apresentam maiores possibilidades de configuração e mobilidade quando comparadas àquelas na multicamada (VAN DER BERG, 1981).

Quando K se aproxima do valor unitário não se observa distinção entre a água líquida e a água presente na multicamada, e o modelo GAB se reduz ao modelo BET, que considera as características da água na multicamada iguais às da água líquida.

$X_{m}$ é conhecido como valor de monocamada e representa uma medida da disponibilidade de sítios ativos para absorção de água pelo material (VAN DER BERG, 1981).

Quirijns (2005a) descreve o significado físico do parâmetro $X_{m}$ como o número de moléculas na monocamada ou, simplesmente, o conteúdo de umidade da monocamada.

A análise qualitativa dos parâmetros GAB torna possível a avaliação do comportamento da umidade adsorvida pela combinação dos valores de $\mathrm{C}_{\mathrm{g}} \mathrm{e} \mathrm{K}$, conforme descrito na Tabela 1.
Tabela 1 - Combinação da magnitude dos parâmetros $\mathrm{C}_{\mathrm{g}}$ e $\mathrm{K}$ e o correspondente mecanismo de adsorção/dessorção.

\begin{tabular}{cccc}
\hline$M o \approx M u$ & $M o \approx M u$ & $M o \neq M u$ & $M o \neq M u$ \\
$\approx L i q$ & $\neq L i q$ & $\neq L i q$ & $\approx L i q$ \\
\hline $\mathrm{C}_{\mathrm{g}} \approx 1$ & $\mathrm{C}_{\mathrm{g}} \approx 1$ & $\mathrm{C}_{\mathrm{g}}>>1$ & $\mathrm{C}_{\mathrm{g}}>>1$ \\
$\mathrm{~K} \approx 1$ & $\mathrm{~K}<<1$ & $\mathrm{~K} \ll<1$ & $\mathrm{~K} \approx 1$ \\
\hline
\end{tabular}

Fonte: QUIRIJNS et al. (2005b).

\subsection{Calor isostérico líquido e entropia de adsorção/dessorção \\ Um importante parâmetro}

termodinâmico obtido através das isotermas para várias temperaturas é o calor isostérico, que mede a energia das forças de ligação entre as moléculas de água e o sólido (ALMUHTASEB, McMINN e MAGEE, 2002).

Sua medida fornece informações sobre a natureza do mecanismo de adsorção/dessorção e auxilia no processo de determinação dos tipos de água no adsorvato (IGLESIAS e CHIRIFE, 1976).

A diferença entre a quantidade de energia requerida para remover a água de um material e a energia requerida para a vaporização da água líquida na mesma temperatura e pressão do material é definido como calor isostérico líquido $\left(\Delta \mathrm{H}_{\mathrm{is}}\right)$ e pode ser calculado utilizando a equação de ClausiusClapeuron, descrita na Equação 2.

$$
\Delta H_{i s}=-\Re \cdot\left(\frac{\partial \ln a_{w}}{\partial T^{-1}}\right)_{X}
$$

Segundo Quirijns et al.(2005b) essa relação é mais adequada para análises qualitativas sobre a natureza de $\Delta \mathrm{H}_{\mathrm{is}}$, pois seus valores são calculados através de uma série de manipulações algébricas, conduzindo a erros consideráveis, além daqueles já associados às próprias medidas de atividade de água.

Além disso, a Equação 2 leva em conta que $\Delta \mathrm{H}_{\mathrm{is}}$ é invariável com a temperatura. Nesse aspecto os autores alertam que os resultados obtidos para $\Delta \mathrm{H}_{\mathrm{is}}$ representam um 
valor médio no intervalo de temperaturas avaliado.

Quirijns et al. (2005a), Quirijns et al. (2005b) e Van der Berg (1981) sugerem a utilização apenas dos dados de dessorção, desconsiderando possíveis efeitos de histerese, para a determinação de $\Delta \mathrm{H}_{\mathrm{is}}$. Segundo os autores a dessorção representa melhor os fenômenos como um todo. Além disso os autores argumentam que o estudo de isotermas visa, sobretudo, fornecer informações práticas para processos de secagem, portanto, um processo de dessorção.

A entropia de adsorção/dessorção é a medida da diferença de entropia entre a água adsorvida e a água líquida para processos de adsorção e vice-versa, no caso da dessorção. Ela pode ser calculada através das relações descritas nas Equações 3 e 4 (RAO, RIZVI e DATTA, 2005).

$\Delta G_{i s}=\Re T \ln a_{w}$

$\Delta S_{A / D}=\frac{\Delta H_{i s}-\Delta G_{i s}}{T}$

Considerando $\Delta \mathrm{H}_{\mathrm{is}}$ independente da temperatura, substituindo a Equação 3 na Equação 4, obtemos a expressão para o cálculo de $\Delta \mathrm{S}_{\mathrm{A} / \mathrm{D}}$ pela Equação 5 .

$\ln a_{w}=\frac{\Delta H_{i s}}{\mathfrak{R}} \cdot \frac{1}{T}-\frac{\Delta S_{A / D}}{\mathfrak{R}}$

\section{MATERIAIS E MÉTODOS}

\subsection{Preparação das amostras}

Para a medida das isotermas de adsorção, amostras de cevada da variedade Brau foram colocadas em estufa a $60^{\circ} \mathrm{C}$ pelo período de sete dias até atingirem teor de umidade de $1,7 \%$ b.s..

Nas isotermas de dessorção, amostras de cevada da variedade Brau foram submetidas à reumidificação por submersão em água a $15^{\circ} \mathrm{C}$ pelo período de $24 \mathrm{~h}$, seguidas por um período de descanso de $2 \mathrm{~h}$ dispostas em monocamada, a temperatura de $25^{\circ} \mathrm{C}$, de forma que atingissem teor de umidade de cerca de $0,6 \mathrm{~kg} \cdot \mathrm{kg}^{-1}$.

A umidade das amostras foi realizada em triplicata por um analisador de umidade da marca Denver.

\subsection{Metodologia para a determinação das isotermas}

Empregou-se o método gravimétrico estático utilizando soluções saturadas sob condições de temperatura constante.

Neste método a temperatura e a umidade relativa do ambiente são mantidas constantes até que a massa das amostras permaneça constante entre duas pesagens sucessivas.

Frascos foram preenchidos cada um com uma solução saturada conforme os dados disponíveis na Tabela 2.

Tabela 2 - Umidades relativas para as soluções saturadas em diferentes temperaturas.

\begin{tabular}{ccccc}
\hline Composto & $15^{\circ} \mathrm{C}$ & $20^{\circ} \mathrm{C}$ & $30^{\circ} \mathrm{C}$ & $50^{\circ} \mathrm{C}$ \\
\hline $\mathrm{KOH}$ & 0,09 & 0,08 & 0,07 & 0,06 \\
$\mathrm{CH}_{3} \mathrm{COOK}$ & 0,21 & 0,21 & 0,20 & 0,19 \\
$\mathrm{MgCl}_{2}$ & 0,33 & 0,33 & 0,32 & 0,31 \\
$\mathrm{~K}_{2} \mathrm{CO}_{3}$ & 0,43 & 0,43 & 0,43 & 0,43 \\
$\mathrm{Mg}\left(\mathrm{NO}_{3}\right)_{2}$ & 0,55 & 0,55 & 0,48 & 0,47 \\
$\mathrm{NaNO}_{2}$ & 0,67 & 0,65 & 0,62 & 0,60 \\
$\mathrm{NaCl}$ & 0,77 & 0,76 & 0,75 & 0,75 \\
$\mathrm{KCl}$ & 0,86 & 0,85 & 0,82 & 0,80 \\
\hline
\end{tabular}

Fonte: BARROZO (1995).

No topo de cada frasco foi acoplado um recipiente perfurado contendo a amostra de cevada em monocamada de forma que a solução não entrasse em contato com a amostra.

A massa de amostra foi determinada por balança analítica com precisão de 0,001g. Pesou-se cerca de 2,500g de cevada para cada 
recipiente. Os ensaios foram realizados em câmara com controle de temperatura da marca Binder.

As amostras permaneceram nessas condições por 30 dias. Após esse período as massas foram determinadas e as amostras recolocadas na estufa por mais 7 dias, para a realização de nova pesagem a fim de se determinar se não houve variação da massa.

\section{RESULTADOS E DISCUSSÕES}

\subsection{Isotermas de adsorção e dessorção}

A Figura 1 trás a representação gráfica das isotermas de adsorção e dessorção de sementes de cevada nas temperaturas de $15^{\circ} \mathrm{C}$, $20^{\circ} \mathrm{C}, 30^{\circ} \mathrm{C}$ e $50^{\circ} \mathrm{C}$.

Figura 1 - Isotermas de adsorção e dessorção para sementes de cevada inteiras em várias temperaturas.



Fonte: Acervo pessoal.

Através do gráfico da Figura 1 foi possível constatar que os dados referentes às isotermas de dessorção apresentaram maiores valores de umidade de equilíbrio quando comparados com seus respectivos correspondentes para adsorção em todos os valores de atividade de água.

Esse comportamento indica a presença de histerese, e segundo Quirijns et al. (2005b) tal efeito deve ser evitado para os cálculos das propriedades termodinâmicas.
Van der Berg (1981) sugere que, nos ensaios de adsorção, partindo de um material rico em amido e com teor de umidade reduzido, há a possibilidade de sítios de adsorção não estarem disponíveis na sua totalidade.

Já na dessorção, uma vez que o material foi previamente submetido a condições de umidade superiores às condições de equilíbrio numa dada temperatura e umidade ambiente, tais sítios estariam disponíveis.

Esse efeito pode também ser observado nos dados representados na Figura 1, onde a histerese diminuiu consideravelmente na medida em que a atividade de água aumentou, com consequente aumento na umidade de equilíbrio.

Também foi possível observar que, para isotermas de temperaturas diferentes, o valor da umidade de equilíbrio diminui com o aumento de temperatura para uma mesma atividade de água.

Tal constatação era esperada, uma vez que o aumento de temperatura, para uma mesma atividade de água, favorece a saída de água do material.

Partindo do que foi sugerido por Quirijns et al (2005a) e Quirijns et al (2005b), utilizou-se os dados das isotermas de dessorção para análise. Esses dados foram comparados com os reportados na literatura por Vermuganti (1980). Tal comparação está ilustrada no gráfico da Figura 2.

Em comparação com os dados reportados por Vermuganti (1980), foi possível observar que, para atividades de água inferiores a 0,4 , houve uma maior concordância com os dados experimentais.

Acima desse valor foi possível observar que, para uma mesma atividade de água e temperatura, os dados reportados na literatura apresentam maiores valores de umidade de equilíbrio.

Ainda que as variedades de cevada sejam distintas, é possível que esse efeito 
ocorra em função da temperatura na qual as espécies foram cultivadas.

As cevadas utilizadas por Vermuganti (1980) foram cultivadas nos Estados Unidos, que apresentam temperatura média anual sensivelmente menor do que a temperatura da região onde as cevadas da variedade Brau foram cultivadas no Brasil.

Essa diferença de temperatura pode acarretar numa mudança na conformação interna do tecido celular e das cadeias de amido, o que poderia causar a diminuição do número de sítios de adsorção nas cevadas cultivadas em temperaturas mais elevadas (BEGON, HARPER e TOWNSEND 2007).

Figura 2 - Comparação entre as isotermas de dessorção do presente estudo com aquelas descritas por Vermuganti (1980).



Fonte: Acervo pessoal.

\subsection{Análise dos coeficientes do modelo GAB}

Os dados experimentais para as isotermas de dessorção foram ajustados pelo modelo GAB. O ajuste está representado no gráfico da Figura 3.

Além disso, para efeito de comparação, os dados fornecidos por Vermuganti (1980) foram submetidos ao ajuste pelo modelo $\mathrm{GAB}$ a fim de se comparar a análise termodinâmica para ambos os conjuntos de dados.

Os valores dos parâmetros GAB para cada temperatura juntamente com os coeficientes de ajuste estão apresentados na
Tabela 3 para os dados experimentais e para os dados fornecidos pela literatura.

Figura 3 - Ajustes do modelo GAB às isotermas de dessorção de água em sementes de cevada.

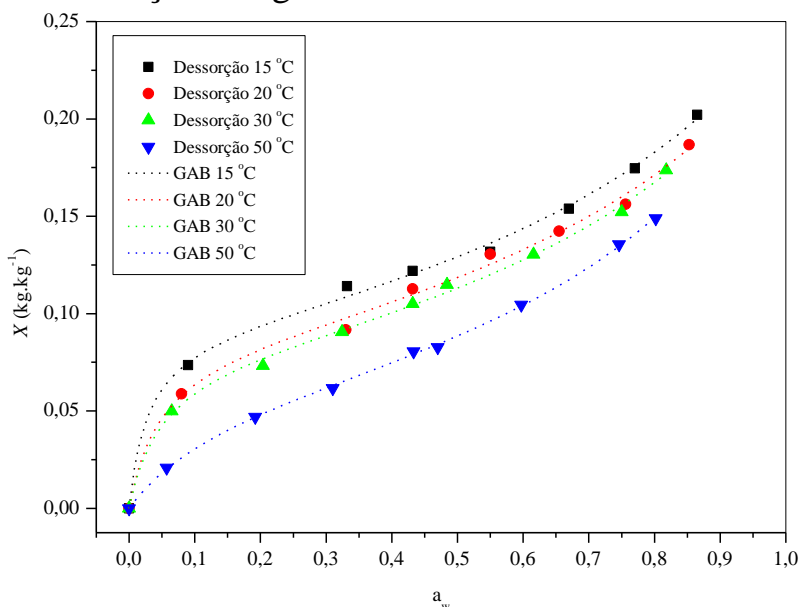

Fonte: Acervo pessoal.

Conforme observado no gráfico da Figura 3, o modelo GAB ajustou satisfatoriamente os dados experimentais para as quatro temperaturas avaliadas no presente estudo.

Tabela 3 - Coeficientes do modelo GAB para isotermas de cevada em várias temperaturas (experimentais e da literatura).

\begin{tabular}{|c|c|c|c|c|}
\hline & \multicolumn{4}{|c|}{ Temperaturas } \\
\hline & \multicolumn{2}{|c|}{$15^{\circ} \mathrm{C}$} & \multicolumn{2}{|c|}{$20^{\circ} \mathrm{C}$} \\
\hline & Exp & $\mathrm{V}$ & Exp & $\mathrm{V}$ \\
\hline$X_{m}$ & 0,092 & 0,076 & 0,086 & 0,070 \\
\hline $\mathrm{C}_{\mathrm{g}}$ & 55,1 & 38,0 & 33,0 & 29,0 \\
\hline $\mathrm{K}$ & 0,63 & 0,80 & 0,64 & 0,81 \\
\hline \multirow[t]{3}{*}{$\mathrm{R}^{2}$} & 0,997 & 0,988 & 0,994 & 0,986 \\
\hline & \multicolumn{2}{|c|}{$30^{\circ} \mathrm{C}$} & \multicolumn{2}{|c|}{$50^{\circ} \mathrm{C}$} \\
\hline & Exp & $\mathrm{V}$ & Exp & V \\
\hline$X_{m}$ & 0,080 & 0,067 & 0,067 & 0,057 \\
\hline $\mathrm{C}_{\mathrm{g}}$ & 30,0 & 26,2 & 9,35 & 20,21 \\
\hline $\mathrm{K}$ & 0,67 & 0,82 & 0,73 & 0,85 \\
\hline $\mathrm{R}^{2}$ & 0,997 & 0,986 & 0,999 & 0,983 \\
\hline
\end{tabular}

Fonte: Acervo pessoal.

Através da Tabela 3 foi possível observar coeficientes de determinação superiores a 0,99 para todos os casos. 
Cabe ressaltar que o modelo GAB também foi eficiente no ajuste dos dados fornecidos por Vermuganti (1980).

Entretanto, é importante reportar que no trabalho de Vermuganti (1980), o autor não explicita seus dados experimentais, mas os calcula utilizando o modelo proposto por Chung e Pfost.

Isso poderia explicar os menores coeficientes de ajuste observados para as regressões aplicadas aos dados da literatura frente aos experimentais, ainda que seu valor se aproxime da unidade.

Considerando o significado físico do parâmetro $X_{m}$ discutido por Quirijns (2005a), podemos observar sua diminuição com o aumento de temperatura.

Esse efeito pode ser explicado considerando que, na medida em que o sistema dispõe de maior conteúdo energético, mais moléculas passam a apresentar propriedades compatíveis com aquelas da multicamada, diminuindo, portanto, o valor de $X_{m}$ quando a temperatura aumenta.

Outro efeito que ocorre com o aumento da temperatura, segundo Quirijns (2005b), é que uma diminuição no valor de $X_{m}$ pode acontecer devido à redução do número de sítios ativos para a ligação com a água devido às mudanças estruturais provocadas no material pelo aumento de temperatura.

A literatura frequentemente admite como constante os valores de $X_{m}$, como no trabalho de Kiranoudis et al. (1993).

Entretanto, Quirijns et al. (2005a) esclarecem que, para uma melhor precisão e coerência física dos valores dos demais parâmetros do modelo $\mathrm{GAB}, X_{m}$ não deve ser considerado constante para temperaturas distintas.

Através dos dados da Tabela 3 também foi possível avaliar qualitativamente o efeito conjugado dos parâmetros $\mathrm{C}_{\mathrm{g}}$ e $\mathrm{K}$ a fim de se determinar as classes de águas presentes no material.
De modo semelhante ao reportado por Quirijns et al (2005b) para cilindros de amido, os ajustes do modelo GAB para as isotermas de cevada apresentou valores de $\mathrm{C}_{\mathrm{g}}$ bem maiores do que a unidade, e valores de $\mathrm{K}$ menores do que a unidade para toda a faixa de temperaturas.

Segundo os autores, e conforme ilustrado na Tabela 1 , isso indica que as moléculas de água estão organizadas em uma monocamada, com moléculas fortemente ligadas aos seus sítios de adsorção e uma multicamada, na qual as moléculas de água apresentam propriedades consideravelmente diferentes em comparação com da água líquida na mesma temperatura e pressão.

Quanto maior o valor de $\mathrm{C}_{\mathrm{g}}$ mais fortemente ligadas as moléculas da monocamada se encontram. Considerando que $\mathrm{C}_{\mathrm{g}}$ diminui com a temperatura, essa análise explica, também, o decréscimo nos valores de $X_{m}$, de forma que as moléculas se encontram cada vez menos ligadas aos seus sítios na monocamada na medida em que a temperatura aumenta.

$\mathrm{Na}$ medida em que a temperatura aumenta, foi possível avaliar um aumento nos valores de $\mathrm{K}$.

Esse resultado é fisicamente coerente, considerando que um aumento na temperatura aumenta o efeito entrópico sobre o sistema, de forma que as moléculas de água apresentem maiores possibilidades de configuração e mobilidade.

A influência da temperatura nos parâmetros $\mathrm{C}_{\mathrm{g}}$ e $\mathrm{K}$ pode ser representada por expressões do tipo Arrhenius através de parâmetros com significado físico.

Infelizmente, a determinação desses parâmetros adicionais, relativos a essas expressões, não foi possível, uma vez que os ajustes não foram satisfatórios de tal forma que permitissem tal análise.

Sendo possível, essa análise permitiria uma comparação com os valores calculados para o calor isostérico através da Equação 5. 
Entretanto, segundo Quirijns (2005a), ainda que não haja precisão nos valores, sua análise qualitativa é de grande utilidade para determinação dos tipos de água presentes no material e pode contribuir significativamente para a melhoria dos processos de secagem.

Aplicando a mesma análise aos dados fornecidos por Vermuganti (1980), foi possível observar concordância com a análise feita para os dados experimentais obtidos no presente trabalho.

\subsection{Determinação de $\Delta H_{\text {is }}$ e $\Delta S_{D}$}

Através dos dados experimentais e daqueles fornecidos por Vermuganti (1980) utilizou-se o modelo GAB para calcular valores de atividade de água como função da temperatura para valores específicos de umidade de equilíbrio.

Utilizando-se a Equação 5, foi possível calcular os valores de $\Delta \mathrm{H}_{\text {is }}$ e $\Delta \mathrm{S}_{\mathrm{D}}$, apresentados nos gráficos das Figuras 4 e 5 .

Conforme previsto, os valores de $\Delta \mathrm{H}_{\text {is }}$ foram positivos, em concordância com $\mathrm{o}$ caráter endotérmico da dessorção.

Foi possível observar que $\Delta \mathrm{H}_{\text {is }}$ diminuiu significativamente com o aumento do teor de umidade após atingir um valor máximo para um teor de umidade de equilíbrio de 0,04 $\mathrm{kg} . \mathrm{kg}^{-1}$, de aproximadamente $2250 \mathrm{~J} / \mathrm{g}$, mantendo-se aproximadamente constante para teores de umidade menores.

Quirijns et al. (2005b) afirmam que isso é um indicativo das forças de atração intermoleculares entre os sítios de adsorção e as moléculas de vapor de água. Segundo os autores, para conteúdos de umidade na região da monocamada, a água é firmemente ligada ao material, correspondendo às maiores energias de interação.

Na medida em que a umidade aumenta, os sítios de adsorção mais ativos energeticamente tornam-se ocupados e iniciase então a ocupação daqueles com menor energia, o que resulta em uma diminuição no
$\Delta \mathrm{H}_{\text {is }}$, que se aproxima do zero para teores de umidade elevados.

Nessas condições, o calor total de adsorção é igual ao calor de vaporização e as moléculas de água no adsorvato se comportam de maneira semelhante energeticamente às da água líquida.

Pode-se observar também, segundo a Figura 4, que o comportamento de $\Delta \mathrm{H}_{\text {is }}$ está de acordo com a análise dos coeficientes do modelo GAB.

A presença de uma monocamada com moléculas fortemente ligadas pode ser observada pelo valor máximo de $\Delta \mathrm{H}_{\text {is }}$. A partir daí, o decréscimo nos valores de $\Delta \mathrm{H}_{\mathrm{is}}$ indica a presença de uma multicamada de tal forma que a umidade nessa região caminhe para valores energéticos correspondentes aos da água líquida.

Figura 4 - Valores de $\Delta \mathrm{H}_{\mathrm{is}}$ para sementes de cevada.

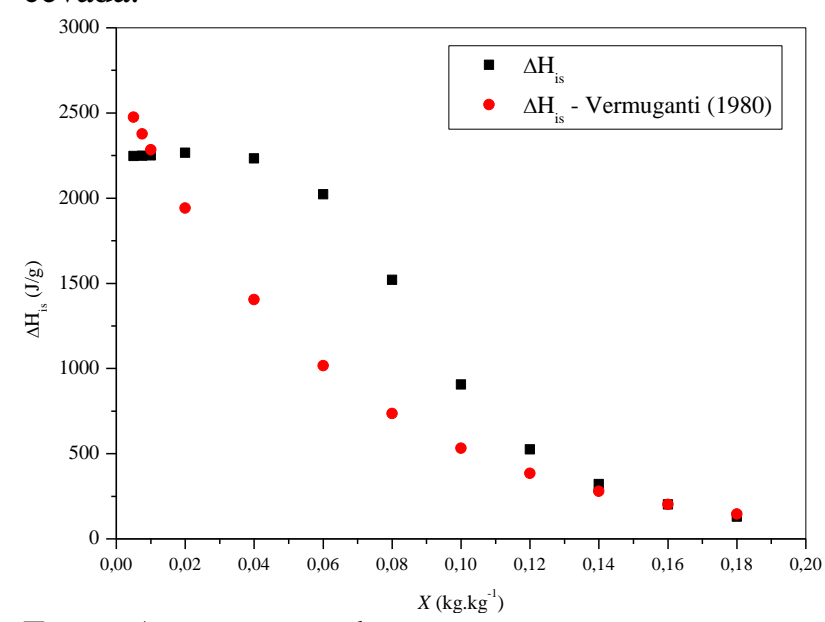

Fonte: Acervo pessoal.

A diferença no comportamento de $\Delta \mathrm{H}_{\mathrm{is}}$ obtido para o presente estudo em comparação com os calculados para os dados de Vermuganti (1980) ocorreu devido ao ajuste de seus dados experimentais pelo modelo de Chung e Pfost.

Segundo Quirijns et al. (2005b) a manipulação algébrica de dados termodinâmicos resulta em discrepâncias significativas nas análises realizadas tanto 
pelo modelo GAB quanto pelos cálculos de $\Delta \mathrm{H}_{\text {is }}$.

Nesse caso, a representação dos dados da literatura apresentou uma inconsistência física, não apresentando diferença de comportamento entre a região de monocamada e a região de multicamada.

O mesmo pode ser observado para os valores de $\Delta S_{D}$ apresentados na Figura 5 .

Figura 5 - Cálculo de $\Delta \mathrm{S}_{\mathrm{D}}$ para sementes de cevada.

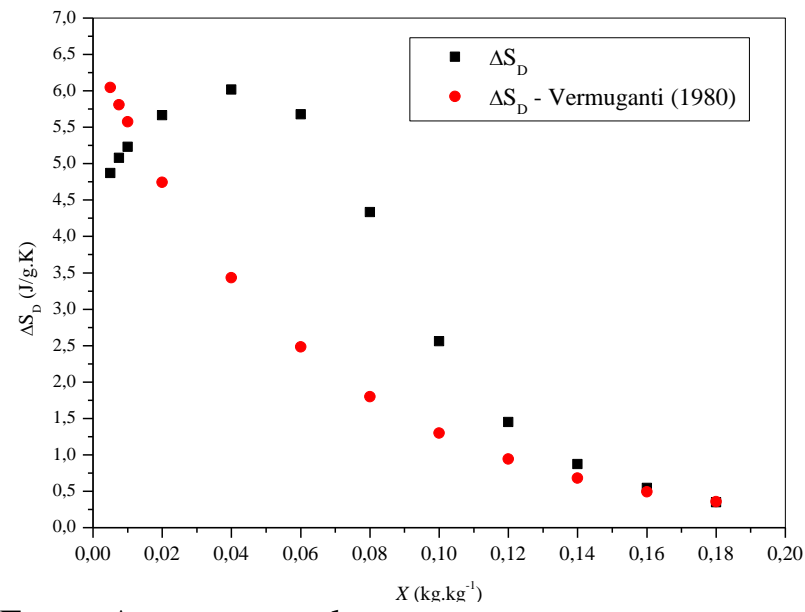

Fonte: Acervo pessoal.

Observou-se coerência nos valores positivos para $\Delta \mathrm{S}_{\mathrm{D}}$. Esse comportamento era esperado uma vez que, na medida em que a umidade aumenta na monocamada a entropia da água diminui, considerando que os sítios são preenchidos e os graus de liberdade das moléculas de água diminuem até que toda a monocamada seja preenchida.

Através do gráfico da Figura 5 foi possível identificar o ponto de máxima variação de entropia entre a água líquida e a água adsorvida, de aproximadamente $6,0 \mathrm{~J} / \mathrm{g} . \mathrm{K}$ para um teor de umidade de 0,04 $\mathrm{kg} \cdot \mathrm{kg}^{-1}$.

A partir desse ponto, o decréscimo no valor de $\Delta \mathrm{S}_{\mathrm{D}}$ indica que a entropia da água adsorvida aumenta, devido ao aumento dos graus de liberdade de suas moléculas na região de multicamada até convergir para o valor de entropia da água líquida, de forma que $\Delta S_{\text {des }}$ se aproxima de zero para teores de umidade elevados.

Outro aspecto relevante foi observado considerando que os valores máximos de $\Delta \mathrm{H}_{\mathrm{is}}$ e $\Delta S_{D}$ ocorrem no mesmo ponto, no teor de umidade de $0,04 \mathrm{~kg} \cdot \mathrm{kg}^{-1}$.

Os valores de $X_{m}$ obtidos pelo modelo $\mathrm{GAB}$, apresentou valores maiores do que o valor de umidade de equilíbrio obtido pelo cálculo de $\Delta \mathrm{H}_{\mathrm{is}}$ e pelo cálculo de $\Delta \mathrm{S}_{\mathrm{D}}$. Ainda que dentro da mesma ordem de grandeza, essa discrepância ocorre pois na análise do modelo GAB a umidade da monocamada é dependente da temperatura e esse efeito desaparece no cálculo do calor isostérico líquido, uma vez que trata-se de valores médios, conforme o que foi descrito por Quirijns et al. (2005b).

De forma semelhante ao observado para $\Delta \mathrm{H}_{\text {is }}$, os valores da literatura para $\Delta \mathrm{S}_{\text {des }}$ não permitiram uma análise termodinâmica satisfatória.

Isso sugere que a presença de modelos empíricos para o ajuste de dados referentes às isotermas de equilíbrio disponíveis na literatura, ainda que sejam eficazes na previsão do comportamento da umidade de equilíbrio para uma dada atividade de água, descaracterizam o comportamento termodinâmico do sistema quando as análises são aplicadas para a região da monocamada.

\section{CONCLUSÕES}

Frente ao exposto foi possível concluir que as isotermas de adsorção e dessorção determinadas no presente estudo encontramse em concordância com as disponíveis na literatura para a mesma faixa de temperaturas.

Além disso, a análise dos parâmetros do modelo GAB, do calor isostérico e da entropia de dessorção foram adequados para uma análise qualitativa da descrição das classes de água presentes no sistema.

Foi possível concluir que, para sementes de cevada, há a formação de uma 
monocamada de água fortemente ligada e uma multicamada, onde a água apresenta características distintas daquelas apresentadas para a água líquida na mesma temperatura e pressão do sistema.

\section{NOMENCLATURA}

$X$ - umidade de equilíbrio [ $\mathrm{kg} \cdot \mathrm{kg}^{-1}$ ]

$X_{m}$ - umidade da monocamada [ $\left.\mathrm{kg} \cdot \mathrm{kg}^{-1}\right]$

$\mathrm{C}_{\mathrm{g}}$ - parâmetro entálpico do modelo $\mathrm{GAB}$

$\mathrm{K}$ - parâmetro entrópico do modelo GAB

$\mathrm{a}_{\mathrm{w}}$ - atividade de água

Mo - monocamada

$\mathrm{Mu}$ - multicamada

Liq - água líquida

$\Delta \mathrm{H}_{\text {is }}$ - calor isostérico líquido $[\mathrm{J} / \mathrm{g}]$

$\Delta \mathrm{S}_{\mathrm{D}}$ - entropia de dessorção [J/g.K]

$\Delta \mathrm{G}_{\text {is }}$ - energia livre de Gibbs $[\mathrm{J} / \mathrm{g}]$

$\Re$ - constante dos gases [J/mol.K]

$\mathrm{T}$ - temperatura $[\mathrm{K}]$

Exp - experimental

V - Vermuganti (1980)

\section{REFERÊNCIAS}

QUIRIJNS, E. J.; VAN BOXTEL, A, J.; VAN LOON, W. K.; VAN STRATEN, G. An Improved Experimental and Regression Methodology for Sorption Isotherms. Journal of the Science of Food and Agriculture, n.85, p.175-185, $2005 \mathrm{a}$.

QUIRIJNS, E. J.; VAN BOXTEL, A, J.; VAN LOON, W. K.; VAN STRATEN, G. An Sorption Isotherms, GAB Parameters and Isosteric Heat of Sorption. Journal of the Science of Food and Agriculture, n.85, p.1805-1814, 2005b.

KIRANOUDIS, C. T.; MAROULIS, Z. B.; TSAMI, E.; MARINOS-KOURIS, D. Equilibrium Moisture Content and Heat of Desorption of Some Vegetables. Journal of Food Engineering, v.20, p.55-74, 1993.
KUNZE, W. Technology Brewing and Malting. Berlin: Versuchs und Lehranstalt für Brauerei in Berlin (VLB), 2004.

VERMUGANTI, G. R. Grains Properties Affecting Storage and Drying . 1980, p.39. Tese - Kansas State University, Manhattan, Kansas.

ANDERSON, R. B. Modification of the Brunauer, Emmett and Teller Equation. Journal of American Chemical Society, v.68, n.4, p.686-691, 1946.

RAO, M. A.; RIZVI, S. S. H.; DATTA, A. K Engineering Properties of Foods. Boca Raton: Taylor and Francis Group, 2005.

VAN DER BERG, C. Vapous Soluption Equilibria and Other Wather-Starch Interactions; a Physico-chemical Approach. 1981, p.201. PhD Tese Wageningen Agricultural University, Wageningen.

AL-MUHTASEB, A. H.; McMINN, W. A.; MAGEE, T. R. Moisture Sorption Isotherm Characteristics of Food Products: A Review. Trans IChemE, v.80, p.118-128, 2002.

IGLESIAS, H. A.; CHIRIFE, J. Isosteric Heats of Water Vapour Sorption on Dehydrated Foods. Part I: Analysis of the Differential Heat Curves. Lebens Wiss Technol, v.9, p.116-122, 1976.

BARROZO, M. A. S. Transferência de Calor e Massa entre o Ar e Sementes de Soja em Leitos Deslizantes e Escoamento Cruzado. 1995. Tese - Universidade Federal de São Carlos, São Carlos.

BEGON, M., HARPER, J. L. TOWNSEND, C. R. Ecologia: de Indivíduos a Ecossistemas. Editora Artmed, p.752, 2007. 